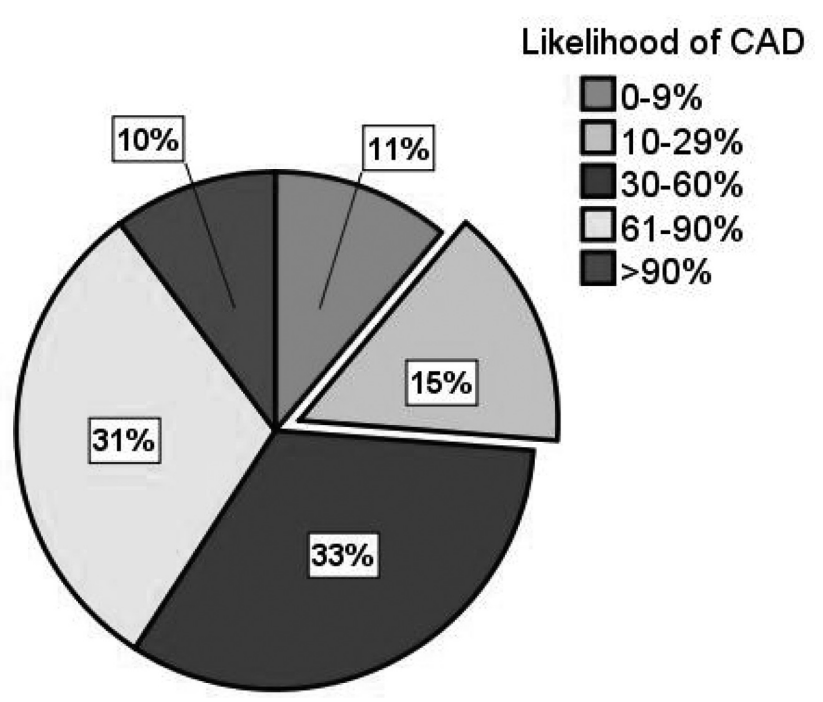

Abstract 133 Figure 1 Estimated likelihood of coronary artery disease according to the NICE guideline (CG95)

\section{NON-INVASIVE INTERROGATION OF MYOCARDIAL DISARRAY IN HYPERTROPHIC CARDIOMYOPATHY}

${ }^{1}$ Laura-Ann McGill*, ${ }^{1}$ Pedro Ferreira, ${ }^{1}$ Andrew Scott, ${ }^{2}$ Sonia Nielles-Vallespin, 'Philip Kilner, ${ }^{1}$ Ranil De Silva, 'David Firmin, ${ }^{1}$ Dudley Pennell. ${ }^{1}$ Royal Brompton Hospital; ${ }^{2}$ National Institute of Health; *Presenting Author

\subsection{6/heartjnl-2016-309890.134}

Introduction Hypertrophic cardiomyopathy (HCM) is an inheritable cardiomyopathy characterised by left ventricular hypertrophy (LVH). In contrast to other aetiologies of $\mathrm{LVH}$, the HCM myocyte microstructure displays disarray, the ability to detect disarray non-invasively could therefore assist the diagnosis in equivocal cases.

In vivo Diffusion tensor imaging (DTI) is a novel cardiovascular magnetic resonance technique, which exploits the fact that intra-myocardial water diffusion reflects the shape and orientation of the underlying microstructure. DTI can characterise the myocardial microstructure with novel parameters: Myocyte orientation can be depicted by the helical angle gradient (HAG); the degree of myocardial organisation can be assessed by fractional anisotropy (FA); and mean diffusivity (MD) quantifies the ease of myocardial water passage. We sought to determine whether DTI could detect myocardial micro-structural differences compared with patients with hypertension and healthy controls.

Methods We recruited 25 patients with HCM, 13 patients with HTN and 14 healthy, age matched controls for DTI at 3T. Three short axis mid-ventricular slices were acquired during the systolic pause, with a diffusion-weighted stimulated echo sequence, as previously described. Data was post-processed to calculate global FA, HAG and MD values. Statistical comparison between the 3 cohorts was made with one way ANOVA, followed by students t-tests were ANOVA was significant.

Results There was no significant difference in FA between cohorts ( HCM: $0.44 \pm 0.05$, HTN: $0.47 \pm 0.06$ and Control: $0.46 \pm 0.05, \mathrm{p}=0.20)$. MD was greater in HCM compared to controls (ANOVA p $=0.002, \mathrm{HCM} 1.07 \pm 0.13$ vs Controls: $\left.0.91 \pm 0.13 \times 10-3 \mathrm{~mm}^{2} \mathrm{~s}^{1}, \mathrm{p}<0.01\right)$, however there was no difference in MD between HCM and HTN (HCM: $1.07 \pm 0.13$ vs HTN: $0.97 \pm 0.14 \times 10-3 \mathrm{~mm}^{2} \mathrm{~s}^{1}, \mathrm{p}=$ 0.15). The HAG was less in HCM compared to both HTN and Controls (HCM: $-6.2 \pm 1.0$, HTN: $-7.4 \pm 0.9$ and Controls: $-8.6 \pm 0.8, \mathrm{p}<0.01)$. The HAG was plotted against maximal wall thickness for all patients combined: correlation coefficient $0.723, \mathrm{p}<0.001$.

Summary DTI was unable to detect a significant disarray in HCM via fractional anisotropy. Explanations include the limited resolution of our sequence, the small study sample and potential regionality of disarray. The higher MD observed in HCM compared to controls may be attributable to a greater extracellular volume as a result of fibrosis. Technical factors such as myocardial strain and heterogeneity of SNR may also have contributed. The HAG was correlated with maximal wall thickness, with reduction in the rate of angular change as the wall thickness increases.

Conclusion DTI provides novel myocardial tissue characterisation, however in its current form it is unable to detect disarray in HCM. Further assessment following technical development is required.

\section{DETECTING PROGRESSION OF DIFFUSE INTERSTITIAL FIBROSIS IN ALSTROM SYNDROME}

${ }^{1}$ Shanat Baig*, ${ }^{2}$ Nicola Edwards, ${ }^{2}$ Boyang Liu, ${ }^{2}$ Manvir Hayer, ${ }^{2}$ Charlotte Dawson, ${ }^{2}$ Tarekegn Geberhiwot, ${ }^{2}$ Richard Steeds. 'University Hospital of Birmingham NHS Trust; ${ }^{2}$ University Hospital Birmingham NHS Trust; *Presenting Author

\subsection{6/heartjnl-2016-309890.135}

Introduction Alstrom Syndrome (ALMS) is a rare inherited disorder caused by a mutation in the ALMS1 gene. The syndrome is a multi-system disorder with exaggerated features of the metabolic syndrome and although rare, provides a monogenic model for end-organ fibrosis and as a paradigm for the effects of severe metabolic syndrome. Adults with ALMS have a high risk of death from heart failure in their twenties due to a cardiomyopathy which (in the small post-mortem series available) is characterised by coarse fibrosis on histology. Our previous work has identified expansion of the extracellular space (ECV) consistent with diffuse interstitial fibrosis in over half of asymptomatic ALMS patients compared to controls. The aim of this study was to investigate the longitudinal change in ECV and assess the impact on ventricular structure and function.

Methods A prospective longitudinal cohort study of patients attending the national service for ALMS at the Centre for Rare Disease in Birmingham from 2012. At referral and on annual follow up, all subjects underwent comprehensive LV and RV assessment with cardiac MRI (CMR 1.5T Siemens Avanto). The presence of diffuse interstitial myocardial fibrosis was assessed using native myocardial T1 relaxation mapping and extracellular volume (ECV) in the LV septum (MOLLI) using cvi42 (Circle Cardiovascular Imaging). Coarse replacement fibrosis was assessed using standard late gadolinium enhancement imaging.

Results In total 14 patients (male gender 71\%, age $28 \pm$ 8years) had baseline and follow up data (median 1.7 [1.1-2.8] years). CMR data is presented in Table 1 . The native LV myocardial T1 values and ECV were increased in the septum at basal and mid levels at follow up. Left ventricular mass increased $\left(54 \pm 9 \mathrm{~g} / \mathrm{m}^{2}\right.$ vs. $\left.62 \pm 12 \mathrm{~g} / \mathrm{m}^{2}\right)$ but with a reduction in septal myocardial intracellular volume (ICV $0.74 \pm$ 0.06 vs. $0.68 \pm 0.04, p<0.05)$ suggesting ECV expansion rather than myocyte hypertrophy was the driver. There were no differences in LV or RV volumes or RVEF. Four patients 
Abstract 135 Table 1 Baseline and Follow-up CMR data

\begin{tabular}{lll}
\hline & Baseline & Follow-up \\
\hline LV Septal T1 (ms) & $952(80)$ & $1040(1070)^{* *}$ \\
LV Basal septal T1 (ms) & $953(63)$ & $1042(76)^{* *}$ \\
LV Mid septal TI (ms) & $949(68)$ & $1033(58)^{* *}$ \\
Septal ECV & $0.26(0.06)$ & $0.32(0.04)^{*}$ \\
LV EDVi $\left(\mathrm{ml} / \mathrm{m}^{2}\right)$ & $57(10)$ & $56(10)$ \\
LVESV $\left(\mathrm{ml} / \mathrm{m}^{2}\right)$ & $20(7)$ & $20(8)$ \\
LVEF $(\%)$ & $65(8)$ & $67(5)$ \\
LV Mass index & $54(9)$ & $62(12)$ \\
RVEDV $\left(\mathrm{ml} / \mathrm{m}^{2}\right)$ & $57(10)$ & $56(12)$ \\
RVESV $\left(\mathrm{ml} / \mathrm{m}^{2}\right)$ & $23(6)$ & $21(6)$ \\
RVEF $\left(\mathrm{ml} / \mathrm{m}^{2}\right)$ & $61(7)$ & $63(7)$ \\
\hline
\end{tabular}

Mean (SD), ** $p<0.01$

had LGE; two patients had focal at RV insertion points LGE and two patients had mid-wall LGE in the basal infero-lateral segments.

Conclusion ALMS is associated with increases in ECV and progressive change in T1 values over time that reflects progression of diffuse interstitial fibrosis in asymptomatic adults. Cross-sectional studies have identified ECV as a biomarker of cardiovascular "vulnerability" but longitudinal tracking has the potential to highlight those at greatest risk.

\section{BEYOND THE AORTA: EXPERIENCES OF NEUROVASCULAR IMAGING IN LOEYS-DIETZ AND VASCULAR EHLERS DANLOS SYNDROME}

Carolyn Smith*, Sarju Mehta, Nigel Burrows, Peter Martin, Rosemary Rusk. Addenbrookes Hospital; *Presenting Author

\subsection{6/heartjnl-2016-309890.136}

Introduction The vascular complications of inherited syndromes Loeys-Dietz (LDS) and Vascular Ehlers Danlos (vEDS) lead to significant morbidity and mortality. Current recommendations advocate head to pelvis screening for LDS, and to a lesser extent for vEDS as complications typically involve medium to large arteries. We reviewed the practice in our aortopathy Inherited Cardiac Conditions Clinic (AICC). In our institution these patients are managed by a multi-specialist approach including cardiologists, neurologists, vascular surgeons, chest physicians, dermatologists and geneticists.

Methods Patients were identified through current attendance at AICC or discussion at the linked multidisciplinary team (MDT) meeting. Electronic notes and imaging were reviewed to determine clinical details, genetic diagnosis, screening offered and (neuro) vascular complications.

Results 36 patients were identified. Of these: 14 had a genetic diagnosis of vEDS, 8 a genetic diagnosis of LDS, 4 were excluded (alternate genetic diagnosis e.g. Marfan's) and the remaining 10 had a suggestive clinical phenotype without a typical LDS/vEDS gene mutation and were reviewed alongside the LDS/vEDS population. Screening was performed with separate CT angiograms (arch to carotids and arch to iliacs) to obtain adequate image quality.

Two patients had clinical neurovascular events (spontaneous dissection). To date neurovascular imaging has been offered to 27 of the 32 patients $(82 \%)$. Of these, two patients declined screening, leading to $78 \%$ of the patient group being studied (25 of 32 ).
Neurovascular involvement of all patients screened reached $52 \%$ (4 vEDS, 4 LDS, 5 other) including a high proportion of the patients (5 of 6 screened) without genetic LDS/vEDS diagnosis (Tables 1, 2; Figure 1). Significant neurovascular pathology was identified in the absence of aortic pathology, including one LDS patient with normal aortic dimensions and morphology, a finding not commonly reported in the literature.

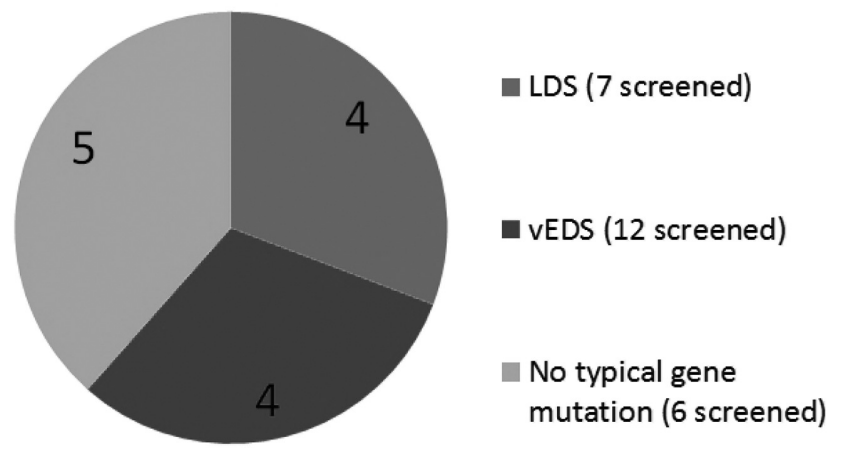

Abstract 136 Figure 1 Neurovascular involvement by diagnosis

Conclusions This is a small population of patients, but the neurovascular involvement rate is high. We would recommend that head to pelvis screening is considered for all LDS and vEDS patients, including those with normal aortic dimensions and morphology. Furthermore, this data implies that patients with a suggestive clinical phenotype should be offered the same screening protocols. It also emphasises the importance of a multi-specialist approach to these patients and their management should not be confined to cardiology alone. Once identified, the specialist MDT is key in making decisions on further surveillance and treatment threshold.

\section{PARADOXICAL EMBOLISM RISK INCREASES WITH ATYPICAL RIGHT ATRIAL BLOOD FLOW IN THE PRESENCE OF A PATENT FORAMEN OVALE}

${ }^{1}$ Victoria Stoll ${ }^{*},{ }^{1}$ Aaron Hess, ${ }^{1}$ Oliver Rider, ${ }^{1}$ Hayley Harvey, ${ }^{1}$ Alex Pitcher, ${ }^{1}$ Margaret Loudon, ${ }^{1}$ Malenka Bissell, 'Stefan Neubauer, ${ }^{2}$ Oliver Ormerod, 'Saul Myerson. 'OCMR, University of Oxford; ${ }^{2}$ Oxford University Foundation Trust; *Presenting Author

\subsection{6/heartjnl-2016-309890.137}

Abstract 136 Table 1 Positive neurovascular imaging results in LDS/vEDS; (ICA, internal carotid artery; TS Trans Sinus; AA Ascending Aorta)

\begin{tabular}{llll}
\hline Diagnosis & Age & Neurovascular & Aorta (cm) \\
\hline LDS & 77 & Left ICA aneurysm multiple vessel ectasia & Dilated TS 4.7 AA 3.8 \\
LDS & 61 & Right ICA aneurysm, tortuosity & Normal TS 3.6 AA 2.4 \\
LDS & 53 & Left middle cerebral artery ectasia & Operated: AA graft \\
LDS & 29 & Right ICA dilatation (?previous dissection) & Operated: AA graft \\
vEDS & 35 & Left ICA pseudoaneurysm & Normal TS 2.4 AA 2.4 \\
vEDS & 38 & Right ICA dilatation (?previous dissection) & Normal TS 2.8 AA 2.3 \\
vEDS & 31 & Bilateral ICA aneurysm and dissection & Dilated TS 3.9 AA 3.1 \\
vEDS & 43 & Left vertebral artery pseudoaneurysm & Normal AA 3.3 \\
\hline
\end{tabular}

